\title{
What is the best Cardiovascular Risk Score for the Brazilian Population?
}

\author{
Bruno Ferraz de Oliveira Gomes ${ }^{1,2}{ }^{\circledR}$ and Gláucia Maria Moraes de Oliveira2 ${ }^{(\mathbb{C}}$ \\ Rede D'Or São Luiz,' Rio de Janeiro, RJ - Brazil. \\ Universidade Federal do Rio de Janeiro, ${ }^{2}$ Rio de Janeiro, $R J$ - Brazil. \\ Editorial referring to the article: Reliability between Cardiovascular Risk Assessment Tools: A Pilot Study
}

Although the cardiovascular disease mortality rates in Brazil still reach high numbers, they have decreased significantly in recent years. In the early 1990s, the country presented approximately 350 deaths per 100000 inhabitants per year, and the most recently published rates were reduced to just over 200 deaths per 100000 inhabitants. Mortality rates due to coronary artery disease are higher in men: between 1990 and 2017, the annual mortality rate due to coronary artery disease was approximately 100 deaths per 100000 inhabitants. Disability-adjusted life years (DALYs) went from more than 6000 years per 100000 inhabitants in the 1990s to just over 4000 years per 100000 inhabitants in the past decade. ${ }^{1}$

Strategies for measuring cardiovascular risk have changed clinical practice by promoting effective preventive measures that reduce the occurrence of major cardiovascular events and improve quality of life. Among them, the most commonly used are simple clinical criteria, clinical prediction scores, imaging examinations, and biomarkers. The clinical application of a cardiovascular risk score should be assessed for its ability to affect the therapeutic management and prognosis of individuals. A risk prediction model must be evaluated in several subsequent phases, such as the initial concept, its prospective validation in independent populations, the incremental information provided in relation to the currently available models, the confirmation of its effects in modifying the clinical conduct and prognosis of patients, and its cost-effectiveness. ${ }^{2}$

\section{Keywords}

Cardiovascular Diseases/mortality; Risk factors; Demography; Epidemiology; Population Characteristics, Propensity Score; Socioeconomic Factors; Ethnic Groups.
A patient may be allocated in different categories depending on the cardiovascular risk score used, but therapeutic measures should not differ substantially. Therefore, the health care team should discuss which type of patient would benefit the most from the information provided by the risk prediction model. A current line of thought states that patients at intermediate risk need to be reclassified. However, in the primary prevention of cardiovascular disease, intermediate-risk patients already receive the same type of preventive care as high-risk patients. ${ }^{3}$

The Brazilian Cardiovascular Prevention Guideline of the Brazilian Society of Cardiology ${ }^{4}$ recommends the Framingham global risk score, which includes a 10-year estimate of coronary and cerebrovascular events, peripheral arterial disease, or heart failure. The guideline also considers that cardiovascular additional risk factors, significant atherosclerosis, or subclinical atherosclerosis should lead to a risk reclassification regardless of the Framingham risk score. The early identification of patients at higher risk could allow for several interventions aimed at reducing the occurrence of cardiovascular events, especially by implementing population measures in a multidisciplinary approach. ${ }^{4}$

The Framingham studies in Massachusetts started at the end of last century and are based on higher cardiovascular mortality rates, with an overestimation of current risk in different populations worldwide, as is the case in Europe and Brazil. Furthermore, this score does not take into account factors that are currently considered relevant, such as body mass index and obesity, ethnicity, socioeconomic factors, family history, presence of comorbidities such as concomitant kidney disease, physical inactivity, and the prevalence of cardiovascular disease among different populations. More recent studies suggested

Mailing Address: Gláucia Maria Moraes de Oliveira

Universidade Federal do Rio de Janeiro - R. Prof. Rodolpho P. Rocco, 255 - 8. Andar - Sala 6, UFRJ. Postal Code 21941-913, Cidade Universitária, RJ - Brazil

E-mail: glauciam@cardiol.br, glauciamoraesoliveira@gmail.com

DOI: https://doi.org/10.36660/ijcs.20200272 
adjustments to recalibrate this score, but were not able to sufficiently improve its performance. However, they can better predict the risk for a given continent, such as the Systematic Coronary Risk Evaluation (SCORE) that was designed for the European population. In order to extend the risk prediction, some models are able to estimate 30 -year or lifelong risks, but they require validations in different populations. ${ }^{3}$

Marasciulo et al. ${ }^{5}$ compared the use of SCORE with the Framingham risk score in a Brazilian population. ${ }^{5}$ The SCORE tool used data from more than 250000 individuals in 12 European countries to predict cardiovascular death in 10 years, with different models for countries with high or low incidence of cardiovascular disease (SCOREHigh and SCORE-Low, respectively). ${ }^{6}$ The study was conducted at a university hospital with 51 patients aged between 40 and 65 years and without a diagnosis of cardiovascular disease. Most patients had low levels of income and education, and the population presented a high prevalence of hypertension, diabetes, overweight, and dyslipidemia. Framingham, SCORE-High, and SCORE-Low scores were applied to these patients, and a higher proportion of high-risk classifications was observed when using the Framingham score. Finally, the Framingham score showed a good correlation with the SCORE-High but not with the SCORE-Low, suggesting that the SCORE-High may be a good alternative in the Brazilian population. ${ }^{5}$

No risk score has ever been designed for the Brazilian population, leading physicians to use scores that were created using populations with different characteristics.

\section{References}

1. Oliveira GMM, Brant LCC, Polanczyk, CA, Biolo A, Nascimento BR Malta DC. Estatística Cardiovascular - Brasil 2020. Arq Bras Cardiol. 2020; 115(3):308-439

2. Karmali KN, Persell SD, Perel P, Lloyd-Jones DM, Berendsen MA, Huffman MD, Cochrane Heart Group. Risk scoring for the primary prevention of cardiovascular disease Cochrane Database Syst Rev. 2017 Mar; 2017(3): CD006887. Published online 2017 Mar 14. doi: 10.1002/14651858.CD006887.pub4

3. Sarah Zheng, Benjamin Lubin, Rhoda Au, Joanne M. Murabito, Emelia J. Benjamin, Michael Shwartz. Advantages of Continuous-Valued Risk Scores for Predicting Long-Term Costs: The Framingham Coronary Hear Disease 10-Year Risk Score Adv Geriatr Med Res. Author manuscript available in PMC 2019 Aug 23.Adv Geriatr Med Res. 2019; 1(1): e190004 Published online 2019 Jun 6. doi: 10.20900/agmr20190004
In addition to the traditional risk factors, economic and social factors also interfere with cardiovascular mortality ${ }^{7}$, hence scores based on European populations are expected to underestimate the real cardiovascular risk in Brazilian people.

Patients with diabetes are at high cardiovascular risk regardless of the score used. ${ }^{4}$ In the study by Marasciulo et al. $^{5}$ people with diabetes represented $27.5 \%$ of the sample. The Framingham score classified $71.4 \%$ of these patients as high-risk. In comparison, the SCORE-High and SCORE-Low identified $35.7 \%$ and $21.4 \%$ of high-risk patients, respectively; this demonstrated the superiority of the Framingham score in characterizing risk in patients with diabetes.

The small sample size represents an important limitation of the described study, as well as the lack of follow-up for verifying the actual occurrence of cardiovascular events. The identification of the best score to be applied to the Brazilian population must be based on a long-term follow-up that allows the observation of the real rate of occurrence of events. Nevertheless, the study presents an alternative regarding the Framingham risk score that deserves to be further studied in the Brazilian population.

In conclusion, several risk prediction models have independent predictive values, but this criterion is not enough to guarantee clinical utility. An increased prognostic capacity, the prediction of therapy benefits, and clinical efficacy are criteria required when assessing the predictive value of a risk score to be used in the Brazilian population.

4. Précoma DB, Oliveira GMM, Simão AF, Dutra OP, Coelho OR, Izar $\mathrm{MCO}$, et al. Atualização da Diretriz de Prevenção Cardiovascular da Sociedade Brasileira de Cardiologia - 2019. Arq Bras Cardiol. 2019; 113(4):787-891

5. Marasciulo RC, Stamm AMNF, Garcia GT, Marasciulo ACE, Rosa AC, Remor AAC et al . Reliability between Cardiovascular Risk Assessment Tools: A Pilot Study. Int. J. Cardiovasc. Sci. 2020; 33(6):618-626.

6. Conroy RM, Pyöräla K, Fitzgerald AP, Sans S, Menotti A, De Backer G, et al. Estimation of ten year risk of fatal cardiovascular disease in Europe: the SCORE project. Eur Heart J. 2003;24(11):987-1003

7. Kreatsoulas C, Anand SS. The impact of social determinants on cardiovascular disease. Can J Cardiol. 2010;26 Suppl C(Suppl C):8C-13C. doi:10.1016/s0828-282x(10)71075-8 